\title{
Writer Identification of Chinese Handwriting Using Grid Microstructure Feature
}

\author{
Xin Li and Xiaoqing Ding \\ State Key Laboratory of Intelligent Technology and Systems \\ Department of Electronic Engineering, Tsinghua University, Beijing 100084, China \\ \{lixin, dxq\}@ocrserv.ee.tsinghua.edu.cn
}

\begin{abstract}
This paper proposes a histogram-based feature to Chinese writer identification. It is called grid microstructure feature. The feature is extracted from the edge image of the real handwriting image. The positions of edge pixel pairs are used to describe the characteristics in a local grid around every edge pixel. After global statistic, the probability density distribution of different pixel pairs is regarded as the feature representing the writing style of the handwriting. Then the similarity of two handwritings is measured with the improved weighted visions of some original metric. On the HIT-MW Chinese handwriting database involving 240 writers, the best Top-1 identification accuracy is $95.0 \%$ and the Top-20 accuracy reaches $99.6 \%$.
\end{abstract}

Keywords: Writer identification, Chinese handwriting, grid microstructure feature, improved weighted metric.

\section{Introduction}

Automatic writer recognition has been promoted by some valuable researches in recent years. Western handwriting recognition technology has been experimented on large handwriting database and has shown the practical effectiveness. But eastern writer recognition still has some challenges. The basic reason is that western writing (like English) is alphabetic writing while eastern writing (like Chinese) is ideographic writing. English letters are simple and Chinese characters have complex stroke crossing. Western writing has lots of ink connection between individual letters. But eastern writing, no matter Chinese, Japanese or Korean, is composed of characters in separate block structure. The existing methods of Chinese writer recognition mainly divide into two classes: the identical character based text-dependent approaches and the text-independent approach based on texture analysis [1]. Wang [2] proposed a method using directional element features and linear transform, and got rather high accuracy by combining several characters. However, there are tens of thousands of different characters in Chinese and even three to six thousands characters are in common use. If handwritings have only few identical characters even none, the text-dependent methods cannot work. Moreover, the text-independent metheds has no limits to text content. M. Yoshimura [3] counted relative frequencies of some model patterns from Japanese sentences to identify writers. Some texture analysis based 
methods are also introduced in Chinese writer recognition. Zhu [4] used multichannel Gabor features to Chinese handwritings. Wavelet-based HMT models were utilized by He [5]. These methods use character patching to create texture blocks. But actually the order of character arrangement influences the texture and the complex structures of Chinese characters enhance the influence. So the methods based on texture analysis do not perform as well on Chinese handwritings as on English handwritings. Bulacu and Schomaker 6] proposed a number of very effective histogram-based features for western writer recognition.

In this paper, we propose a histogram-based feature, the grid microstructure feature, for Chinese writer identification. The idea of the feature comes from the contour-hinge feature, which outperforms the other textural and allographic features in 6]. Differently, the proposed feature is generated from a size-adjustable grid and focuses on the positions of edge pixel pairs, while the contour-hinge feature examines the directions of two linked edge fragments. To improve the performance of writer identification, we try different distance metrics for similarity measurement and introduced the improved weighted metrics. The experimental results show the effectiveness of the proposed method. The remaining part of this paper is organized as follows: Section 2 describes the novel histogram-based feature, the grid microstructure feature and the methods of the similarity measurement. Then section 3 introduces the Chinese handwriting database and the experiment process. Section 4 shows and analyzes the results of the experiments. Finally, the conclusions are given in Section 5 .

\section{Grid Microstructure Feature}

The grid microstructure feature is extracted from the edge image of the handwriting image. Edge image is seen as a primary representation of handwritten document. The preprocessing step of our method is to gain the edge image. Real handwritings are scanned to gray images firstly. After binarization using Otsus method, edge detection is carried out. Among many edge detection algorithms, the Sobel operator is used in our implementation. In the edge image, Bulacu et al. saw the two contour fragments extending from an edge pixel as a rotatable hinge and used the direction combination of the fragment pair to describe the small region around the center edge pixel [7]. Maaten fused multi-scale contourhinge features, whose contour fragment length takes different values, to improve the performance [8]. Our proposed feature is to record the positions of some special edge pixel pairs in every local grid, to calculate the appearance probability of different position pairs and to express the writing style by the probability density distribution.

\subsection{Feature Generation}

We use a small part of an edge image, which contains a single handwritten character, to demonstrate the process of feature generation as Fig. 1 shows. We assume that a floating grid is moving along the edge traces in the image 
and its center square will traverse all edge pixels. Assume that the horizontal and vertical distances between the border squares and the center square of the grid are both $L$, so the size of the floating grid is $(2 L+1) \times(2 L+1)$. For the diagrammatic sketch in Fig. 1, $L=4$. Every square position of the grid is marked with a fixed symbol $i_{m}$, where $m$ denotes the bigger distance in the horizontal and vertical distances between the square and the center. For each value of $m$, there are $8 m$ square positions and they are assigned from $0_{m}$ to $(8 m-1)_{m}$. In Fig. 1, all square positions except the center are signed with $0_{1}, \ldots, 7_{1}, 0_{2}, \ldots, 15_{2}, 0_{3}, \ldots, 23_{3}, 0_{4}, \ldots, 31_{4}$. If there are two edge pixels in some pair of squares, the symbols of the squares are used to record the positions of the edge pixel pair, such as $\left\langle i_{m}, j_{l}\right\rangle$. When the center of the grid is moving along the edge traces, we use $h\left(i_{m}, j_{l}\right)$ to record the occurrence number of edge pixels in each square pair of the local grid. In the beginning, we set $h\left(i_{m}, j_{l}\right)=0$ $(1 \leq m, l \leq L, 0 \leq i \leq 8 m-1,0 \leq j \leq 8 l-1)$. When the grid center is located at a new edge pixel and there are edge pixels in two squares like $i_{m}$ and $j_{l}$, we make

$$
h\left(i_{m}, j_{l}\right)=h\left(i_{m}, j_{l}\right)+1 .
$$

But not all edge pixel pairs have the effectiveness to represent the writing style. Firstly we record the edge pixel pairs which satisfy the set of conditions

$$
\left\{\begin{array}{l}
1 \leq m=l \leq L, i<j \\
i_{m} \text { and } j_{l} \text { are the edge pixels } \\
k_{m} \text { is not the edge pixel, } i<k<j
\end{array}\right.
$$

Under the conditions, the positions of the edge pixel pairs, which are the same distance away from the grid center, are recorded. We use the continuous distance values $1 \leq m \leq L$ to describe the change of the same edge trace in the local grid, such as $<1_{1}, 6_{1}>$ and $\left.<3_{2}, 11_{2}\right\rangle$. Also the relations between different edge traces are recorded, such as $<11_{2}, 14_{2}>$ and $<4_{3}, 9_{3}>$. For $i<j$, we do not record the pairs like $<31_{4}, 6_{4}>$. Maatten used the implementation to reduce the complexity in his writer identification software 9.

In addition, the extend trend and the turning of a edge trace is more important. A Chinese character always includes much more strokes and has a much finer structure than a western letter. There are many turning points on the edge traces of Chinese characters, such as $\left\langle 14_{2}, 23_{3}\right\rangle$ in Fig.1. Different writers have different writing manners of stroke turnings. We need to record the pairs of pixels which have different distances from the grid center. So we define two other sets of conditions as

$$
\left\{\begin{array}{l}
1 \leq m=l-1 \leq L-1 \\
i_{m} \text { and } j_{l} \text { are the connected edge pixels } \\
i_{m} \text { is the nearest to } j_{l}
\end{array}\right.
$$

and

$$
\left\{\begin{array}{l}
1 \leq m=l-2 \leq L-2 \\
i_{m} \text { and } j_{l} \text { are the connected edge pixels } \\
i_{m} \text { is the nearest to } j_{l}
\end{array}\right.
$$




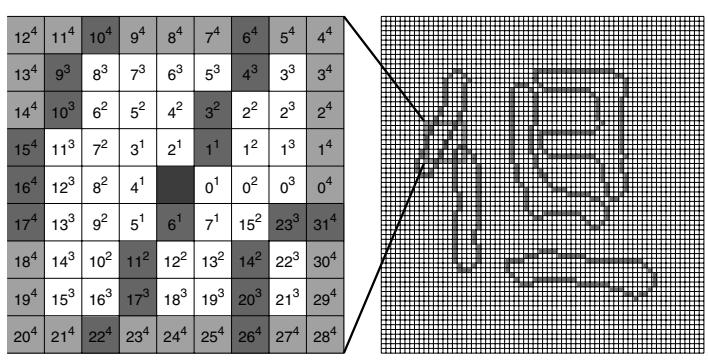

Fig. 1. The example of feature generation when the floating grid lies at a edge pixel. The edge pixel pairs satisfying $\left.C_{1}:\left\langle 1_{1}, 6_{1}\right\rangle,\left\langle 3_{2}, 11_{2}\right\rangle,\left\langle 11_{2}, 14_{2}\right\rangle,<4_{3}, 9_{3}\right\rangle$, $<9_{3}, 10_{3}>, \ldots,<20_{3}, 23_{3}>,<66_{4}, 10_{4}>,<10_{4}, 15_{4}>, \ldots,<26^{4}, 31_{4}>$. The pairs satisfying $C_{2}:\left\langle 1_{1}, 3_{2}\right\rangle,\left\langle 61_{1}, 11_{2}\right\rangle,\left\langle 3_{2}, 4_{3}\right\rangle,\left\langle 11_{2}, 17_{3}\right\rangle,\left\langle 14_{2}, 20_{3}\right\rangle$, $<14_{2}, 23_{3}>,<4_{3}, 64>, \ldots,<23_{3}, 31_{4}>$. The pairs satisfying $C_{3}:<1_{1}, 4_{3}>$, $<6_{1}, 17_{3}>,<3_{2}, 6_{4}>,<11_{2}, 22_{4}>, \ldots,<14_{2}, 31_{4}>$.

Eq. 2 Eq. 3 and Eq. 4 define three sets of conditions. Let $C_{1}, C_{2}$ and $C_{3}$ denote them. In Fig. 1, the positions of the edge pixel pairs which satisfy the three sets of conditions are indicated with $\left\langle i_{m}, j_{l}\right\rangle$ in the caption. We implement Eq. 1 for $h\left(i_{m}, j_{l}\right)$ where $i_{m}$ and $j_{l}$ in accord with these conditions.

After the center of the floating grid has traversed all edge pixels in the edge image, $h\left(i^{m}, j^{l}\right)$ gives the occurrence number of that the edge pixels appear at the square pairs $\left\langle i_{m}, j_{l}\right\rangle$ in local regions. The sum of all occurrence number is $H=\sum i, j, m, l h\left(i_{m}, j_{l}\right)$. Then the appearance probability of the pixel pair $<i_{m}, j_{l}>$ can be calculated by $p\left(i_{m}, j_{l}\right)=\frac{h\left(i_{m}, j_{l}\right)}{H}$. The $2 \mathrm{D}$ probability density distribution is called the grid microstructure feature.

Although each handwriting consists of different characters, every character is made up of some elementary sorts of strokes and their deformations. A writer does not write one sort of strokes just the same every time. The edge pairs will appear at different square positions of the moving grid, even around the same sort of strokes. After global statistic, the appearance probabilities of different pairs are variant. However, the writing style of a writer tends to be uniform and the writing tendencies of different writers are discriminating. The variant probability distribution still reflects the writing tendency of the writer because the writer writes in his common manner unconsciously. Different writers write each sort of strokes with different lengths, different breadths, different angles and different curvatures. These characteristics are all considered and computed by the statistic of the grid microstructure feature.

Fig. 2 and Fig 3 show four Chinese handwritings written by two writers (A and $\mathrm{B}$ ) and their microstructure features under all condition sets, $C_{1}, C_{2}$ and $C_{3}$. Each writer has two handwritings. Fig. 4 gives the diagrams of the absolute feature differences between two handwritings which are written by the same writer or different writers. It is observed intuitively that the difference between handwritings of different writers is distinctly bigger than the difference between handwritings of the same writer. The grid microstructure feature embodies the difference of writing style. 


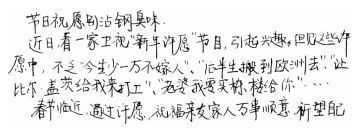

(a) Handwriting $A 1$ of $A$.

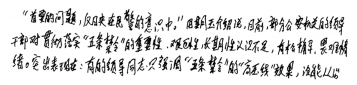

(c) Handwriting $B 1$ of $B$.
心想事成，本无可厚非，然而，热事对金钱财宫占有的表 白，无形中传递着一种不建康的价值取向。其实、美好 的棈神想藉才是人不可或铁的。希望电视节目里的视洞 贺诺别沾来铜臭味。 江西省兴国县陈小兵

(b) Handwriting $A 2$ of $A$.

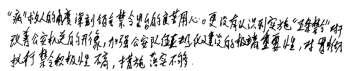

(d) Handwriting $B 2$ of $B$.

Fig. 2. Four handwritings written by two writers

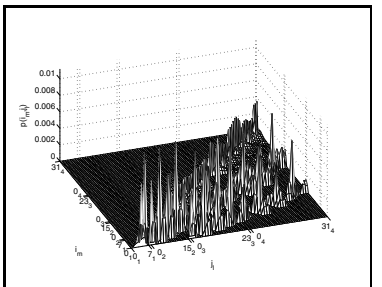

(a) From handwriting $A 1$.

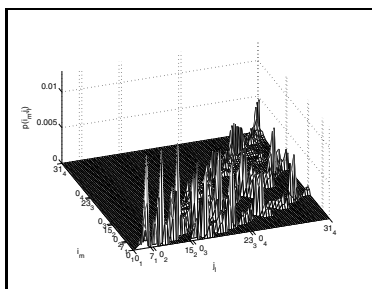

(c) From handwriting $B 1$.

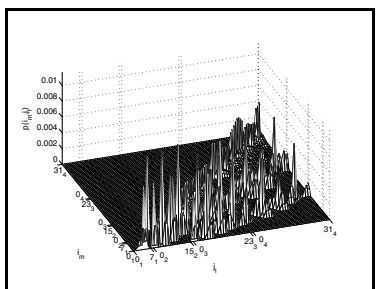

(b) From handwriting $A 2$.

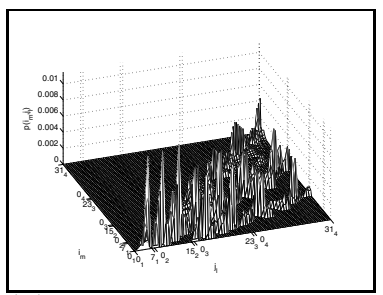

(d) From handwriting $B 2$.

Fig. 3. Grid microstructure features extracted from four handwritings

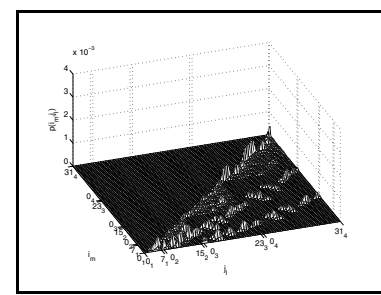

(a) Between $A 1$ and $A 2$.

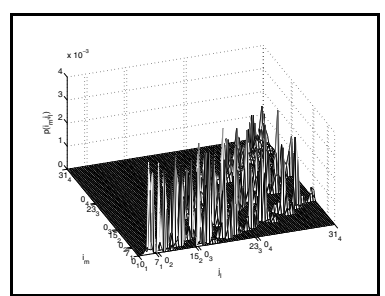

(b) Between $A 1$ and $B 1$.

Fig. 4. The absolute differences between the features of two handwritings 


\subsection{Similarity Measurement}

To compare a handwriting to another, the difference of writing style should be quantified. We need to measure the similarity of two microstructure features. By arranging all probabilities in a high-dimensional vector, the similarity measurement can be transformed into the distance measurement. The most common distance metric is Euclidean metric though it may not be the best. Belongie used the Chi-square metric ( $\chi^{2}$ distance) after extracting shape contexts for object recognition [10. Schomaker and Bulacu also considered that Chi-square metric, in which differences of the low probabilities are weighed more importantly, is better than Euclidean metric and Bhattacharya metric [6]. And Zhu [4] used weighted Euclidean distance measure. They used the standard deviation of handwritings written by the corresponding reference writer to weight the difference of each component. If each reference writer has only one handwriting, the weighted method is disable. However, there are also many different reference writers in writer identification. The reference handwritings of all writer give the range of each feature component. The differences of the components with smaller ranges should be given more weight. So we use the standard deviation of all reference handwritings to weight each component. We define two new distance metrics: the improved weighted Euclidean metric (IWED) and the improved weighted Chisquare metric (IWCD). Assume that there are a query handwriting and $N$ reference handwritings, and their microstructure features are $\mathbf{q}$ and $\mathbf{r}_{n}(1 \leq n \leq N)$. The improved weighted Euclidean distance between $\mathbf{q}$ and $\mathbf{r}_{n}$ is

$$
d_{I W E D}\left(\mathbf{q}, \mathbf{r}_{n}\right)=\sqrt{\sum_{k}\left(\frac{q^{(k)}-r_{n}^{(k)}}{\sigma^{(k)}}\right)^{2}},
$$

and the improved weighted Chi-square distance is

$$
d_{I W C D}\left(\mathbf{q}, \mathbf{r}_{n}\right)=\sum_{k} \frac{\left(q^{(k)}-r_{n}^{(k)}\right)^{2}}{\left(q^{(k)}+r_{n}^{(k)}\right) \sigma^{(k)}},
$$

where $q^{(k)}$ is $k$ th component of feature vector $\mathbf{q}, r_{n}^{(k)}$ is $k$ th component of $\mathbf{r}_{n}$ and $\sigma^{(k)}=\sqrt{\frac{1}{N-1} \sum_{n=1}^{N}\left(r_{n}^{(k)}-m^{(k)}\right)^{2}}, m^{(k)}=\frac{1}{N} \sum_{n=1}^{N} r_{n}^{(k)}$. Then in writer identification, the writer of the reference handwriting corresponding to $\underset{\mathbf{r}_{n}}{\operatorname{argmin}} d\left(\mathbf{q}, \mathbf{r}_{n}\right)$ is considered as the most possible writer of the query handwriting.

\section{Handwriting Database and Experiment Process}

We use a database of handwritten texts, the HIT-MW database, in our experiments. HIT-MW is collected by Artificial Intelligence Lab, Harbin Institute of Technology, China [11. The database is built for Chinese character recognition. The texts in HIT-MW are different and sampled from China Daily. We got the writer information of a part of the database involving 240 writers. Most of the 
writers have only one page of handwritten text. We have to use only one text page for each writer and segment each page into two commensurate parts by manual work. Each part contains about 100 characters and is seen as an independent handwriting. The handwritings $A 1$ and $A 2$ in Fig. 2 also come from the same text page in HIT-MW and so do $B 1$ and $B 2$. In this case, the within-class variability is limited and it helps get higher identification accuracy. However, HIT-MW is the best choice in the public Chinese handwriting databases, because it has a large number of writers and provides the writer information.

We carry out writer identification using the segmented handwritings of 240 writers in HIT-MW. One segmented handwriting of each writer is used as the query handwriting and the other one is used as the reference handwriting. So there is only one handwriting sample for each writer in reference handwriting database. For every query handwriting, the distances between it and all reference handwritings are calculated. The writer of the reference handwriting corresponding to the minimal distance is the first candidate. If the candidate is just the writer of the query handwriting, the identification is correct. The correct rate of all 240 query handwritings is the identification accuracy of our experiment. We also make a longer list of candidates. The correct hit rate increases with the length of the candidate list. When there are $M$ candidates in the list, the correct hit rate is called Top- $M$ recognition accuracy.

\section{Experimental Result}

To begin with, the size of floating grid has influence on the effectiveness of our grid microstructure feature. The information is limited when the grid size is small, and the oversize grid affects the description of local particulars. A best selection of the grid size exists and relates to the scanning resolution of the handwriting samples. HIT-MW samples are all scanned with the resolution 300 dpi. We change the grid size by choosing different values of $L$ in order to get the best grid size. Here the improved weighted Euclidean metric is used. Fig. 5 gives four curves of the correct hit rates when the grid size is changed. The curves are

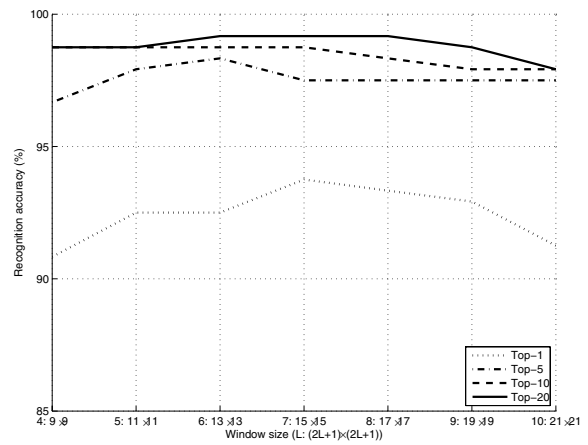

Fig. 5. Accuracy curves of the microstructure feature for different grid sizes 
Table 1. Identification performance of microstructure feature using different distance metrics

\begin{tabular}{|c|c|c|c|c|}
\hline Distance metric & $E D$ & $C D$ & $I W E I$ & $I W C D$ \\
\hline $\begin{array}{l}\text { Top-1 recognition } \\
\text { accuracy }(\%)\end{array}$ & 80.4 & 88.8 & 93.8 & 95.0 \\
\hline
\end{tabular}

Table 2. Recognition accuracies of writer identification using different features

\begin{tabular}{|c|c|c|c|c|}
\hline Features & \multicolumn{4}{|c|}{$\begin{array}{l}\text { Recognition accuracy }(\%) \\
\text { Top-1 Top-5 Top-10 Top-20 }\end{array}$} \\
\hline $\mathrm{CH}$ & 84.6 & 95.4 & 96.7 & 98.3 \\
\hline$M C H$ & 92.5 & 97.1 & 97.5 & 99.2 \\
\hline$G M S^{*}$ & 94.6 & 97.5 & 98.8 & 99.6 \\
\hline$G M S$ & 95.0 & 98.3 & 98.8 & 99.6 \\
\hline
\end{tabular}

Table 3. Identification performance of our method and some other methods

\begin{tabular}{lll}
\hline Method & $\begin{array}{l}\text { Database size } \\
\text { (writer number) }\end{array}$ & $\begin{array}{l}\text { Top-1 recognition } \\
\text { accuracy }(\%)\end{array}$ \\
\hline Our & 240 & 95.0 \\
Zhu's [4] & 17 & 95.7 \\
He's [5] & 500 & 36.4 \\
Yoshimura's [3] & 20 & 94.2 \\
\hline
\end{tabular}

obtained when the length of the candidate list is 1, 5, 10 and 20. We find that when $L$ takes value from 6 to 8, the recognition accuracies are the highest. So we make $L=7$ and choose $15 \times 15$ for the best grid size. In fact, the handwritten characters in the 300 dpi resolution are about from 40 to 70 pixels high. It is reasonable to use the $15 \times 15$ grid to represent the stroke-level information.

Furthermore, similarity measurement is also an important problem. We test four different distance metrics, Euclidean distance (ED), Chi-square distance (CD), improved weighted Euclidean distance (IWED) and improved weighted Chi-square distance (IWCD), by comparison. The microstructure features with $L=7$ are extracted. Table 1 shows the identification performance (Top-1 recognition accuracy) changing with the distance metric. The best result is gotten by the improved weighted Chi-square metric.

Finally, we compare the proposed feature with two existing histogram-based features, the contour-hinge feature ( $\mathrm{CH}$ for short, proposed by Bulacu et al. 7]) and the multi-scale contour-hinge ( $M C H$ for short, suggested by Maaten [8]) in the Chinese handwriting database. We also compare two versions of grid microstructure feature, GMS $S^{*}$ and $G M S$. GMS $S^{*}$ is the feature only satisfying the condition sets $C 1$ and $G M S$ is obtained under the case of all three condition sets. The length of edge fragment in $C H$ is 8 and the value set of fragment length in 
$M C H$ is $2,4,6,8$. The grid size of grid microstructure features is $15 \times 15$. In the comparison, the improved weighted Chi-square distance is computed for similarity measurement. Table 2 gives the Top- 1 , Top- 5 , Top-10 and Top-20 recognition accuracies using the four features. The grid microstructure features outperform the other two features and GMS is better than $G M S^{*}$. It is obvious that our histogram-based feature is more effective for identifying Chinese handwritings.

\section{Conclusions}

For reference, we list some results of the literatures. Table 3 includes the performance of our method and some other text-independent methods for Chinese writer identification and one method for Japanese handwriting]. The recognition accuracy of our method using the grid microstructure feature and the improved weighted Chi-Square metric seems better than the existing text-independent methods for Chinese writer identification. Because of using different databases and different conditions, the results are incomparable. However, Gabor and wavelet features used in the traditional text-independent methods of Chinese writer identification are affected greatly by the normalization and the arrangement of characters in texture blocks. Differently, our histogram-based feature uses original handwriting images and is independent on the order of character arrangement. The grid microstructure feature tries to find out the writing tendency of the writer in local regions. It discriminates the writers using the difference of the tendency. Although the number of Chinese characters is huge and their structures are variant, the sorts of strokes composing characters are limited. If characters in the handwritings are enough to make the appearance percentages of different sorts of strokes balanced, the grid microstructure feature will succeed in denoting the difference of writing style. The effectiveness of our method for Chinese writer identification is proved by the experiments. It is expectable that the feature can be used for multilingual handwritings including western handwritings. As a future work, the effect on the recognition rate caused by the difference of text contents need to be studied. Besides, how to extract writing style information from a smaller number of characters is still a hard problem.

Acknowledgments. This work is supported by National Basic Research Program of China (973 program) (2007CB311004) \& National Natural Science Foundation of China (project 60772049, 60872086).

\section{References}

1. Plamondon, R., Lorette, G.: Automatic Signature Verification and Writer Identification - the State of the Art. Patt. Rec. 22(2), 107-131 (1989)

2. Wang, X., Ding, X., Liu, H.: Writer Identification Using Directional Element Features and Linear Transform. In: 7th International Conference on Document Analysis and Recognition, pp. 942-945. IEEE Press, New York (2003)

\footnotetext{
${ }^{1}$ We do not cite the best result of [3] because it was obtained in the text-dependent case that some query text rows and reference rows have the same text.
} 
3. Yoshimura, M., Yoshimura, I., Kim, H.B.: A Text-Independent Off-line Writer Identification Method for Japanese and Korean Sentences. IEICE Trans. Inf. and Syst. E76-D(4), 454-461 (1993)

4. Zhu, Y., Tan, T., Wang, Y.: Biometric Personal Identification Based on Handwriting. In: 15th International Conference on Pattern Recognition, vol. 2, pp. 797-800. IEEE Press, New York (2000)

5. He, Z., You, X., Tang, Y.: Writer Identification of Chinese Handwriting Documents using Hidden Markov Tree Model. Patt. Rec. 41(4), 1295-1307 (2008)

6. Bulacu, M., Schomaker, L.: Text-Independent Writer Identification and Verification using Textural and Allographic Features. IEEE Trans. on Patt. Ana. and Mach. Inte. 29(4), 701-717 (2007)

7. Bulacu, M., Schomaker, L., Vuurpijl, L.: Writer Identification using Edge-Based Directional Features. In: 7th International Conference on Document Analysis and Recognition, pp. 937-941. IEEE Press, New York (2003)

8. Maaten, L., van der Postma, E.: Improving Automatic Writer Identification. In: 17th Belgium-Netherlands Conference on Artificial Intelligence, pp. 260-266 (2005)

9. WRIDE: Maaten's writer identification software, http://www.cs.unimaas.nl/ 1.vandermaaten/Laurens_van_der_Maaten/Software.html

10. Belongie, S., Malik, J., Puzicha, J.: Shape Matching and Object Recognition using Shape Contexts. IEEE Trans. on Patt. Ana. and Mach. Inte. 24(4), 509-522 (2002)

11. Su, T., Zhang, T., Guan, D.: Corpus-Based HIT-MW Database for Offline Recognition of General-Purpose Chinese Handwritten Text. Int. J. of Doc. Ana. and Rec. 10(1), 27-38 (2007) 